\title{
Análise do componente curricular "Pacientes com Necessidades Especiais" nos cursos de Odontologia do estado do Rio Grande do Norte
}

\author{
Maria Helaynne Diniz Faria*; Francisca Janiele Pinheiro Pereira*; Isabela Pinheiro Cavalcanti Lima**
}

* Estudante, Curso de Odontologia, Universidade do Estado do Rio Grande do Norte

** Docente, Departamento de Odontologia, Universidade do Estado do Rio Grande do Norte

Recebido: 20/01/2021. Aprovado: 24/01/2021.

\begin{abstract}
RESUMO
Este estudo objetivou avaliar a inserção da disciplina de Pacientes com Necessidades Especiais (PNE) na estrutura curricular dos cursos de Odontologia em funcionamento no estado do Rio Grande do Norte (RN). Trata-se de estudo transversal, exploratório e descritivo com abordagem quantitativa. As informações foram coletadas nos sítios web dos cursos. Foram encontrados dez cursos em funcionamento e cinco deles ofertavam a disciplina, sendo apenas um em modo optativo. A nomenclatura da disciplina e o semestre de oferta variou entre as instituições. Dois cursos possuem uma abordagem teórico/prática, um deles clínica e o restante teórica. Conclui-se que apenas as quatro instituições privadas oferecem de forma obrigatória o componente curricular relacionados a PNE durante a graduação, enquanto entre as públicas apenas uma oferta a disciplina, porém, de maneira optativa. Assim, é evidente a necessidade da inserção de conteúdos relacionados à assistência de pacientes especiais nos cursos de graduação em Odontologia do $\mathrm{RN}$, atendendo às Diretrizes Curriculares Nacionais no tangente à formação de um profissional generalista.

Descritores: Pessoas com deficiência. Educação em Odontologia. Estudantes de Odontologia.
\end{abstract}

\section{INTRODUÇÃO}

A abordagem odontológica aos pacientes portadores de necessidades especiais aconteceu pela primeira vez nos Estados Unidos da América, por volta de 1930-1935, quando dois odontopediatras decidiram agrupar aqueles indivíduos que apresentavam determinados desvios dos padrões de normalidade, mais especificamente deficiência física e/ou mental (denominados à época de handicapped) e que, por isso, não poderiam receber tratamento dentário regular. Em 1950, ainda nos Estados Unidos, surgiu um novo conceito em Odontologia: A Odontologia para o Paciente Especial, substituindo o termo excepcional para especial ${ }^{1}$.

Atualmente, no Brasil, são denominados Pessoas com Necessidades Especiais (PNE) indivíduos que possuem alguma alteração ou 
condição, temporária ou definitiva, simples ou complexa, de origem física, mental, biológica, social ou de comportamento e que necessitam de uma abordagem diferenciada e integrativa, seguindo um protocolo específico para seu atendimento ${ }^{2}$.

As Diretrizes Curriculares Nacionais (DCN) dos cursos de Odontologia no Brasil ${ }^{3}$, vigentes desde 2002, assim como sua proposta de atualização aprovada pelo Conselho Nacional de Educação em $2018^{4}$, determinam que o profissional egresso; o cirurgião-dentista, tenha formação generalista, humanística, crítica e reflexiva, capaz de atuar em todos os níveis de atenção à saúde. Além disso, a Odontologia é uma ciência que interage com diversas áreas de atuação, a qual torna relevante a inclusão de Odontologia para Pacientes com Necessidades Especiais (OPNE) nos componentes curriculares das Instituições de Ensino Superior (IES). No entanto, a Lei n. ${ }^{\circ} 9.394$, a qual estabelece as diretrizes e bases da educação à nível nacional, assegura em seu artigo 53 que as IES brasileiras têm autonomia didático-científica para estabelecer a programação curricular de cada curso, cabendo a cada instituição optar por oferecer ou não OPNE em sua estrutura curricular'5.

De acordo com os dados do censo demográfico de 2010, divulgados pelo Instituto Brasileiro de Geografia e Estatística (IBGE), mais de 45,6 milhões de brasileiros possuem algum tipo de deficiência, o que corresponde a cerca de $24 \%$ da população brasileira. O maior percentual de pessoas com pelo menos um tipo das deficiências investigadas (visual, auditiva, mental ou motora) reside no Nordeste do país representando cerca de $27,8 \%$ da população da região. Entre os estados brasileiros, o Rio Grande do Norte (RN) destaca-se com o maior índice de indivíduos com deficiência, aproximadamente $27,7 \%$ dos habitantes do estado $^{6}$.

O tratamento de PNE ainda é considerado um desafio, tanto pela demanda elevada quanto pela escassez de profissionais habilitados, seja no serviço público ou privado. Logo, a condição de saúde bucal desses pacientes torna-se bastante prejudicada, não só pela falta de assistência odontológica, mais sim pela maior prevalência de algumas doenças bucais devido a fatores imunológicos acarretados pela deficiência ${ }^{7}$. Dessa forma, o propósito deste estudo foi analisar a inclusão da disciplina de PNE na estrutura curricular dos cursos de Odontologia em funcionamento no estado do Rio Grande do Norte.

\section{METODOLOGIA}

Trata-se de um estudo transversal de caráter exploratório e descritivo com abordagem quantitativa. Foi dispensada a submissão ao comitê de ética pela forma como se deu a coleta de dados secundários, considerados de domínio público. Os cursos de graduação foram identificados na base de dados oficial e única de informações relativas às IES, no endereço eletrônico emec.mec.gov.br.

Foram incluídos na pesquisa todos os cursos de Odontologia em atividade no estado do RN, no ano de 2019. Optou-se por excluir aqueles que não possuíam um sítio $w e b$ oficial e não forneciam meios de comunicação virtual, assim como os que não apresentavam sua estrutura curricular completa disponível e aqueles que não possuíssem em seu componente curricular a nomenclatura específica de pacientes especiais ou odontologia especial. Tal critério foi proposto com a finalidade de padronização dos dados, uma vez que a nomenclatura OPNE ou Pacientes Especiais possui em sua essência um conceito mais amplo e abrangente, não se restringindo a um grupo específico de pacientes com deficiência ou qualquer outro comprometimento.

Um formulário baseado no estudo de Penha et $\mathrm{al}^{8}$. (2018) foi empregado nesta pesquisa, a fim de facilitar a coleta dos dados, contendo informações sobre as IES (pública ou privada), a existência ou não da oferta de conteúdos 
relacionados a PNE na grade curricular.

Em casos de oferta, coletaram-se as seguintes variáveis: caráter obrigatório ou optativo, nomenclatura, natureza (teórica, clínica ou teórica/prática), semestre no qual era ofertada e carga horária. Os dados desta pesquisa foram tabulados e analisados por meio de estatísticas descritivas.

\section{RESULTADOS}

Foram encontradas sete IES que ofertam o curso de graduação em Odontologia e sete preencheram os critérios de inclusão. Dessas, duas oferecem o curso em cidades diferentes, totalizando dez cursos. Verificou-se que oito cursos $(80 \%)$ são provenientes de instituições privadas e dois (20\%) de públicas.

Dos dez cursos que foram analisados, apenas quatro IES (40\%) - todas elas privadas ofertam a disciplina em caráter obrigatório e um curso público $(10 \%)$ em optativo. No tocante à análise da nomenclatura, natureza, semestre em que é ofertada os resultados mostraram variabilidade, conforme demonstrado na tabela 1 .

Tabela 1. Caracterização dos cursos de Odontologia do RN que possuem o conteúdo de Pacientes Especiais em seu componente curricular, 2019

\begin{tabular}{clcc}
\hline IES & \multicolumn{1}{c}{ Nomenclatura } & Natureza & Semestre \\
\hline Privada & Estágio em Clínica Integrada a Pacientes & Clínica & NI \\
& Especiais & & \\
Privada & Odontologia para Pacientes Portadores de & Teórico/prática & $9^{\circ}$ e $10^{\circ}$ \\
& Necessidades Especiais & & \\
Privada & Odontologia Especial & Teórico/prática & $7^{\circ}$ \\
Pública & Odontologia para Pacientes Especiais & Teórica & NI \\
\hline
\end{tabular}

Foi observado que a carga horária variou de 45 a 160 horas, com média de 89 horas. Em nenhum dos cursos incluídos nesse estudo os conteúdos estavam associados a outras disciplinas, os mesmos funcionavam de maneira independente.

\section{DISCUSSÃO}

A Odontologia para PNE, atualmente pautada em bases científicas, busca uma abordagem ampla e integrada. Assim, reconhecese a importância de práticas clínicas motivadoras durante a graduação, que preparem o futuro profissional visando um atendimento satisfatório destes pacientes, uma vez que é determinado pelas DCN que o perfil do cirurgião-dentista deve ser generalista capaz de atuar em todos os níveis de atenção, o que inclui a atenção ao $\mathrm{PNE}^{9}$.
Estas pessoas estão atingindo idades cada vez mais avançadas em decorrência dos avanços científicos e tecnológicos ${ }^{10}$. A abordagem precoce de agravos bucais nesses indivíduos é um fator importante para se estabelecer uma melhor qualidade de vida. Assim, estratégias de prevenção e promoção de saúde bucal, realizadas por estudantes e profissionais da área se fazem necessárias para o alcance desse objetivo, bem como aumenta a necessidade de profissionais capacitados para a assistência a essa população ${ }^{8}$.

Mudanças nos cursos de graduação em Odontologia vem sendo observadas nos últimos anos, enfatizando a necessidade de formar um profissional com visão humanística, integrativa e capaz de visualizar o indivíduo como um todo, ofertando ao mercado de trabalho um cirurgião- 
dentista eticamente comprometido com a sociedade ${ }^{11}$.

Com a crescente preocupação na formação dos profissionais da área odontológica, foi realizado um estudo em 2005, o qual analisou a oferta da disciplina de pacientes portadores de necessidades especiais pelas universidades do Brasil por meio de um questionário, enviado a 175 instituições cadastradas no Conselho Federal de Odontologia (CFO), no qual apenas 55 participaram da pesquisa e 31 destas ofereciam o conteúdo de pacientes com necessidades especiais na graduação, das quais 22 eram na modalidade obrigatória e nove na modalidade optativa $^{12}$.

Tal estudo corrobora os resultados obtidos nesta pesquisa, das quais entre os dez cursos do $\mathrm{RN}$, quatro dispõe do componente curricular na modalidade obrigatória e apenas uma optativa. Ao contrário dos resultados encontrados em um estudo realizado no estado da Paraíba, em que $100 \%$ dos cursos avaliados, a disciplina foi ofertada em modo obrigatório ${ }^{8}$. Ao mesmo tempo fica a critério da instituição possuir ou não a disciplina de Odontologia para PNE em sua estrutura curricular, uma vez que a Lei federal de n. ${ }^{\circ} 9.394$ assegura autonomia didático-científica para as IES brasileiras ${ }^{5}$. Porém, na medida em que se tornou reconhecida como especialidade pelo $\mathrm{CFO}^{13}$, notou-se um crescimento na oferta dessa disciplina nos cursos de graduação ${ }^{12}$.

Das quatro IES que ofertam a disciplina, duas são de natureza teórico-prática, uma delas é de natureza exclusivamente clínica. Esse método de ensino possibilita o desenvolvimento integral ao acadêmico de graduação, pois a teoria e a prática clínica proporcionam o aprimoramento dos seus conhecimentos quando expostos a realidade da comunidade e do serviço, trazendo benefícios ao discente que se tornará mais qualificado e capaz de desempenhar um importante papel na contribuição do cuidado ao paciente especial $^{9-14}$.

Um estudo realizado por Jacomine et $\mathrm{al}^{15}$. (2018) com graduandos da Faculdade de Odontologia de Bauru (FOB-USP) mostrou que alunos do $2^{\circ}$ ano do curso expressaram a necessidade de conteúdos de PNE na modalidade obrigatória. Um outro estudo realizado com graduandos do último semestre de Odontologia de duas instituições do estado de Sergipe revelou que os acadêmicos relataram a importância e a necessidade de conteúdos curriculares relacionados à pacientes especiais abordados durante a graduação, considerando tema de grande relevância para o futuro profissional ${ }^{9}$. Silva et $\mathrm{al}^{16}$. (2020) destacaram que a oferta da disciplina de OPNE durante a graduação proporciona mais segurança na atuação profissional, além de despertar interesse no atendimento odontológico adequado a esses pacientes.

O presente estudo demonstrou como limitação a ausência de informações completas e oficiais de todas as IES do estado sobre a sua estrutura curricular, o que compromete o processo de escolha dos discentes por uma instituição capaz de despertar seu interesse em estudar conforme as disciplinas ofertadas. Além disso, essas informações deveriam ser de amplo e fácil acesso, contribuindo, por exemplo, no desenvolvimento de estudos científicos que visem melhorias no funcionamento dos cursos de graduação em Odontologia do estado do RN.

Ainda hoje no Brasil há uma lacuna nas estruturas curriculares dos cursos de Odontologia com relação à formação do profissional para atender as pessoas com necessidades especiais, $o$ que enfatiza a proposta de uma revisão das DCN, as quais estão em atualização sob o parecer CNE/CES no: 803/2018, que aguarda homologação do Ministério da Educação; pois os currículos das IES expressam a concepção que se tem sobre saúde, sobre o profissional que se quer 
formar e o papel deste na sociedade.

\section{CONCLUSÃO}

Apenas as quatro IES privadas oferecem o componente curricular relacionados a PNE de forma obrigatória durante a graduação. Entre as duas IES públicas do estado, apenas uma oferta a disciplina, porém, de maneira optativa. Assim, fica evidente a necessidade da inserção de conteúdos relacionados à assistência de pacientes especiais nos cursos de graduação em Odontologia do $\mathrm{RN}$, atendendo às Diretrizes Curriculares Nacionais no tangente à formação de um profissional generalista.

\section{ABSTRACT \\ Analysis of the curricular component "Patients with Special Needs" in Dentistry courses in the state of Rio Grande do Norte}

This study aimed to evaluate the insertion of the Patients with Special Needs (PSN) discipline in the syllabus of Dentistry courses in operation in the state of Rio Grande do Norte (RN). This is a cross-sectional, exploratory and descriptive study with a quantitative approach. The information was collected from the courses' websites. There were ten courses in operation, with five of them offering the discipline, only one in optional mode. The nomenclature of the discipline and the semester of offer varied among the institutions. Two courses have a theoretical/practical approach, one of them clinical and the remaining theoretical. Only the four private institutions offer compulsorily the curricular component related to PSN during graduation, while the only one public institution offers the discipline, but in an optional way. Thus, it is evident the need for the insertion of contents related to the care of special patients in undergraduate Dentistry courses in RN, meeting the National Curriculum Guidelines concerning the training of a generalist professional.

Descriptors: Disabled Persons. Education, Dental. Students, Dental.

\section{REFERÊNCIAS}

(1) Gomes MJ, Caxias FP, Margon CD, Rosa RG, Carvalho RB. A percepção dos docentes do Curso de Odontologia da UFES em relação à necessidade de inclusão da disciplina denominada "Atendimento Odontológico a Pacientes Portadores de Necessidades Especiais". Rev Bras Pesqu Saúde. 2009; 11(1):33-9.

(2) Campos CC, Frazão BB, Saddi GL, Morais LA, Ferreira MG, Setúbal PCO, et al. Manual prático para o atendimento odontológico de Pacientes com Necessidades Especiais. 2ed.Goiás: Universidade Federal de Goiás;2009.

(3) Brasil. Ministério da Educação. Resolução $n^{\circ}$ CNE/ CES 3/2002 de 19 de fevereiro de 2002. Institui as Diretrizes Curriculares Nacionais dos Cursos de Farmácia e Odontologia. Diário Oficial, Brasília, 4 mar 2002, seção 1, p. 10.

(4) Parecer CNE/CES n ${ }^{\circ}$ 803/2018, aprovado em 5 de dezembro de 2018 - Diretrizes Curriculares Nacionais do curso de graduação em Odontologia. [Acesso em: 19 de janeiro de 2021]. Disponível em: http://portal.mec.gov.br/escola-de-gestoresda-educacao-basica/323-secretarias-

112877938/orgaos-vinculados82187207/12991-diretrizes-curricularescursos-de-graduacao.

(5) Brasil. Lei $n^{\circ} 9.394$, de 20 de dezembro de 1996. Estabelece as diretrizes e bases da educação nacional. Brasília: Diário Oficial da União. Seção I, p. 27834-27841.

(6) Censo 2010 - Pessoas com Deficiência / Luiza Maria Borges Oliveira / Secretaria de Direitos Humanos da Presidência da República (SDH/PR) / Secretaria Nacional de Promoção dos Direitos da Pessoa com Deficiência (SNPD) / Coordenação-Geral do Sistema de Informações sobre a Pessoa com 
Deficiência; Brasília: SDH-PR/SNPD, 2012.

(7) Cruz S, Chi DL, Huebner CE. Oral health services within community-based organizations for young children with special health care needs. Spec Care Dentist. 2016; 36(5): 243-53.

(8) Penha ES, Tenório DA, Fonseca FRA, Guênes GMT, Montagna E. Caracterização do componente curricular Odontologia para Pacientes com Necessidades Especiais nos cursos de Odontologia do estado da Paraíba. Rev ABENO. 2018; 18(2):13-19.

(9) Santos MFS, Hora IAA. Atenção odontológica a pacientes especiais: atitudes e percepções de acadêmicos de odontologia. Rev ABENO. 2012;12(2):207-12.

(10) Bonato LL, Lopes AMS, Silva CM, Itner RG, Silva ACH. Situação atual da formação para assistência de pessoas com necessidades especiais nas faculdades de odontologia no Brasil. ClipeOdonto. 2013;5(1):10-5.

(11) Pinheiro FMC, Nóbrega-Therrien SM, Almeida MEL, Almeida MI. A formação do cirurgião-dentista e a promoção de saúde no PSF. Rev Odontol UNESP. 2008;37(1):6977.

(12) Fassina AO, Análise das disciplinas de pacientes portadores de necessidades especiais nas faculdades no Brasil em 2005. [Dissertação de mestrado]. São Paulo:
Faculdade de Odontologia da USP, Universidade de São Paulo;2006.

(13) Conselho Federal de Odontologia. Cursos de Especialização. [Acesso em: 03 de setembro de 2019]. Disponível em: http://www. cfo.org.br.

(14) Mafi A, Moretto C, Teixeira MFN, Saldanha OMFL, Rados ARV. A interdisciplinaridade e seus reflexos na formação do cirurgião-dentista. Rev ABENO. 2017;17(1):62-73.

(15) Jacomine JC, Ferreira R, Sant'Ana ACP, Rezende MLR, Greghi SLA, Damante CA, Zangrando MSR. Saúde bucal e Pacientes com Necessidades Especiais: percepções de graduandos em Odontologia da FOB-USP. Rev ABENO. 2018; 18 (2):45-54.

(16) da Silva RD, Santaella NG, Caminha RDG, Santos PSS. Percepção de estudantes de graduação sobre a importância da disciplina Odontologia para Pacientes com Necessidades Especiais. Rev ABENO. 2020; 20(1):26-32.

\section{Correspondência para:}

Maria Helaynne Diniz Faria e-mail: helaynneodonto@gmail.com Universidade do Estado do Rio Grande do Norte Av. Rio Branco, 725 - Centro 59300-000 Caicó/RN 